\title{
A Perimotor Framework Reveals Functional Segmentation in the Motoneuronal Network Controlling Locomotion in Caenorhabditis elegans
}

\author{
Gal Haspel and Michael J. O’Donovan \\ Laboratory of Neural Control, Section on Developmental Neurobiology, National Institute of Neurological Disorders and Stroke, National Institutes of \\ Health, Bethesda, Maryland 20892
}

\begin{abstract}
The neuronal connectivity dataset of the nematode Caenorhabditis elegans attracts wide attention from computational neuroscientists and experimentalists. However, the dataset is incomplete. The ventral and dorsal nerve cords of a single nematode were reconstructed halfway along the body and the posterior data are missing, leaving 21 of 75 motoneurons of the locomotor network with partial or no connectivity data. Using a new framework for network analysis, the perimotor space, we identified rules of connectivity that allowed us to approximate the missing data by extrapolation. Motoneurons were mapped into perimotor space in which each motoneuron is located according to the muscle cells it innervates. In this framework, a pattern of iterative connections emerges which includes most ( 0.90$)$ of the connections. We identified a repeating unit consisting of 12 motoneurons and 12 muscle cells. The cell bodies of the motoneurons of such a unit are not necessarily anatomical neighbors and there is no obvious anatomical segmentation. A connectivity model, composed of six repeating units, is a description of the network that is both simplified (modular and without noniterative connections) and more complete (includes the posterior part) than the original dataset. The perimotor framework of observed connectivity and the segmented connectivity model give insights and advance the study of the neuronal infrastructure underlying locomotion in C. elegans. Furthermore, we suggest that the tools used herein may be useful to interpret, simplify, and represent connectivity data of other motor systems.
\end{abstract}

\section{Introduction}

The only organism-wide neuronal wiring diagram published to date is that of the nematode Caenorhabditis elegans (White et al., 1986). In the 25 years since its publication, it has attracted wide attention from both computational and experimental neuroscientists (Watts and Strogatz, 1998; Morita et al., 2001; Milo et al., 2002; Sporns and Kötter, 2004; Gray et al., 2005; Chalasani et al., 2007; Bassett et al., 2010; Qian et al., 2011; Varier and Kaiser, 2011). The original dataset was acquired and reconstructed from electron micrographs (White et al., 1986) and recently proofed and annotated (Chen et al., 2006; Varshney et al., 2011). The complete wiring dataset includes 6393 chemical synapses, and 890 gap junctions and 1410 neuromuscular junctions of an adult nematode (White et al., 1986; Altun and Hall, 2008a; Varshney et al., 2011). However, the reconstruction focused on the head and tail ganglia and was assembled from partial reconstructions of

\footnotetext{
Received May 2, 2011; revised July 28, 2011; accepted Aug. 16, 2011.

Author contributions: G.H. designed research; G.H. performed research; G.H. analyzed data; G.H. and M.J.0. wrote the paper.

This work was supported in part by the intramural program of the National Institutes of Health-National Institute of Neurological Disorders and Stroke. We thank David Hall and Beth Chen for discussions of the connectivity database, Sinisa Pajevic for advice on statistics, and Daphne Soares, Kim Hoke, Larry Abbott, and Olaf Sporns for discussions of this manuscript. Some portions of MATLAB code were acquired from MATLAB Central.

The authors declare no competing financial interests.

Correspondence should be addressed to Dr. Gal Haspel, National Institutes of Health-National Institute of Neurological Disorders and Stroke, 35 Convent Drive, MSC 3700, Bethesda, MD 20892. E-mail: haspelg@ninds.nih.gov. DOI:10.1523/JNEUROSCI.2186-11.2011

Copyright $\odot 2011$ the authors $\quad 0270-6474 / 11 / 3114611-13 \$ 15.00 / 0$
}

several different adult animals: one for the nose and the nerve ring, one for nerve ring and anterior cords, one for tail, and one (male) for the posterior nerve cords (White et al., 1986; Varshney et al., 2011) (D. Hall, personal communication). Hence, the anterior portion of the ventral and dorsal nerve cords that contain the locomotor motoneurons was only reconstructed in a single hermaphrodite nematode. Moreover, the nerve cords were reconstructed only halfway along the body, and the data posterior to the vulva are incomplete. The sparse data for the posterior parts of the nerve cords are from a male nematode, and it is unclear how much the neuroanatomy varies between the sexes. Currently, connectivity data are partial or missing for 39 of 302 neurons, including 21 of the 75 locomotor motoneurons. Most publications that discuss the connectivity dataset ignore this fact, and those that mention it do it only briefly.

C. elegans propels itself by the coordinated contraction of muscle cells arranged in four quadrants along the body and neck (Altun and Hall, 2008b). In adult hermaphrodites, 75 of these muscle cells are innervated by 75 motoneurons that have been morphologically separated into eight distinct classes (White et al., 1976, 1986; Chen et al., 2006; Altun and Hall, 2008b). Four classes innervate ventral muscles (12-VA, 11-VB, 6-VC, 13-VD), and four innervate dorsal muscles (11-AS, 9-DA, 7-DB, 6-DD). Most published graphic depictions of the locomotor network (and the complete nervous system) (White et al., 1986; Chalfie and White, 1988; Von Stetina et al., 2006; Sengupta and Samuel, 2009) do not include a depiction of the anterior-posterior (AP) axis, which is crucial for the production of undulatory locomotion. Models 
Table 1. Muscle cell and motoneuron anatomical and perimotor locations

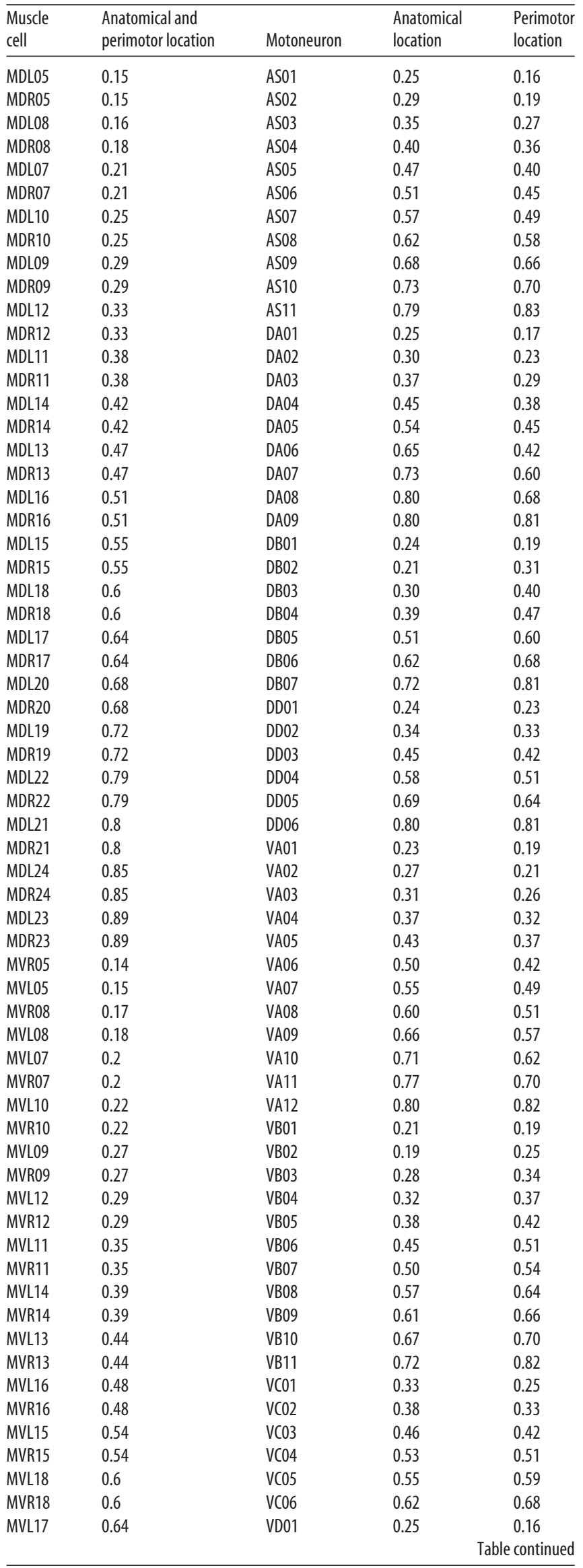

Table 1. Continued

\begin{tabular}{lllll}
\hline $\begin{array}{l}\text { Muscle } \\
\text { cell }\end{array}$ & $\begin{array}{l}\text { Anatomical and } \\
\text { perimotor location }\end{array}$ & Motoneuron & $\begin{array}{l}\text { Anatomical } \\
\text { location }\end{array}$ & $\begin{array}{l}\text { Perimotor } \\
\text { location }\end{array}$ \\
\hline MVR17 & 0.64 & VD02 & 0.26 & 0.21 \\
MVL20 & 0.67 & VD03 & 0.31 & 0.28 \\
MVR20 & 0.67 & VD04 & 0.36 & 0.32 \\
MVL19 & 0.73 & VD05 & 0.42 & 0.39 \\
MVR19 & 0.73 & VD06 & 0.47 & 0.44 \\
MVL21 & 0.77 & VD07 & 0.52 & 0.51 \\
MVR22 & 0.77 & VD08 & 0.59 & 0.54 \\
MVL22 & 0.81 & VD09 & 0.64 & 0.62 \\
MVR21 & 0.81 & VD10 & 0.69 & 0.66 \\
MVL23 & 0.84 & VD11 & 0.74 & 0.70 \\
MVR23 & 0.84 & VD12 & 0.79 & 0.75 \\
MVR24 & 0.89 & VD13 & 0.80 & 0.82 \\
\hline MDL/MDR, & & & & \\
\hline
\end{tabular}

MDL/MDR, Left/right dorsal muscle; MVL/MVR, left/right ventral muscle; AS, DA, DB, DD, VA, VB, VC, VD, motoneurons. Columns 2 and 4 are from supplemental material in the study by Chen et al. (2006).

that incorporate an AP axis (Niebur and Erdös, 1991, 1993; Bryden and Cohen, 2004, 2008; Karbowski et al., 2008) have simplified the network by focusing on forward locomotion, using the same number of motoneurons from each class and omitting motoneuron classes such as AS.

To address the missing data for motoneurons posterior to the vulva, we developed a method for representing motoneuronal connectivity according to the muscles innervated. This method allowed us to group connections according to their function and revealed an unexpected segmented modularity in the organization of the locomotor network. Using this formulation, we could then estimate the missing connections by extrapolation.

\section{Materials and Methods}

The dataset. We obtained the anatomical connectivity data from a publicly available source, WormAtlas (Altun and Hall, 2008a). We also obtained connectivity data for the C. elegans male network (Male Wiring Project, Albert Einstein College of Medicine, website: http://worms. aecom.yu.edu/PHP/male_wiring_project.php), but these data are not yet suitable for our analysis because most of the neuromuscular junctions are not specifically traced to the muscle of origin (S. Emmons, personal communication). The existing connectivity dataset for the hermaphrodite C. elegans is formatted as a table with the following columns: source neuron, target neuron, type of connection, and the number of anatomically observed connections. It has an inherent redundancy in the sense that each gap junction (marked EJ in the dataset) is noted twice, once from each direction. Similarly, chemical synapses are noted at least once as outgoing (marked $\mathrm{S}$ for a simple synapse or marked $\mathrm{Sp}$ where a presynaptic and more than one postsynaptic cells comprise a dyadic synapse) and once for incoming ( $\mathrm{R}$ for simple and $\mathrm{Rp}$ for dyadic). In the original dataset, the total number of synapses is consistent but the number of dyadic versus simple synapses is not consistent (i.e., the designation $S$ and $S p$ add up to the reciprocal $R$ and $R p$, but $S$ does not equal $R$ and $S p$ does not equal $\mathrm{Rp}$ ). As will be explained below, it is not important for our purposes whether a synapse is dyadic. Hence, we first verified that the total number of synapses is indeed consistent and then kept only the record of outgoing synapses ( $\mathrm{S}$ and $\mathrm{Sp}$ ) between motoneurons and all synapses (R, S, Rp, and Sp) to and from non-motoneurons (sensory neurons and interneurons). The number of gap junctions is perfectly reciprocated among neurons, and we considered each gap junction only once. A single self gap junction is included in the dataset (VA08). As explained above, this gap junction was described in a male nematode and was excluded from this study.

Analysis. The analysis program was coded in MATLAB (version R2007b; MathWorks) and is available at MatlabCentral.com or upon request. The program uses the MATLAB statistics toolbox and a MATLAB function "randpermfull" coded by the user "Jos" and available from MATLAB Central. 
Table 2. Of iterated connections that are characteristic of each motoneuron class

\begin{tabular}{|c|c|c|c|c|c|}
\hline Motoneuron class & $\begin{array}{l}\text { Neuromuscular } \\
\text { junctions }\end{array}$ & $\begin{array}{l}\text { Chemical synapses to other } \\
\text { motoneurons }\end{array}$ & Gap junctions with other motoneurons & $\begin{array}{l}\text { Chemical synapses from sensory and } \\
\text { interneurons }\end{array}$ & $\begin{array}{l}\text { Gap junctions } \\
\text { with sensory and } \\
\text { interneurons }\end{array}$ \\
\hline AS & 4 dorsal & $\mathrm{DA}, \mathrm{DD}, \underline{\mathrm{VD}}$ & $\mathrm{DA}, \mathrm{VA}\left(\right.$ posterior $^{a}$ ) & $A V A, A V B, A V D, \underline{A V E}$ & $\underline{\text { AVA }}$ \\
\hline DA & 6 dorsal & DB, DD, VD (1 posterior, 1 anterior) & AS (posterior), VA (anterior) & $\underline{\text { AVA }}$, AVD, AVE HSNR, PVCL, SABVL & $\underline{\text { AVA }}$ \\
\hline DB & 6 dorsal & AS, DD, VD (1 posterior, 1 anterior) & $\underline{\mathrm{DB}}\left(1\right.$ posterior, ${ }^{a} 1$ anterior $\left.{ }^{a}\right), \mathrm{VB}\left(\right.$ anterior $\left.^{a}\right)$ & DVA, PVC, PVR & AVB \\
\hline DD & 6 dorsal & VD & DD (1 posterior, ${ }^{a} 1$ anterior $\left.^{a}\right), \underline{\text { VD }}$ & $\underline{\mathrm{RID}}, \underline{\mathrm{VCO} 1-03}$ & \\
\hline VD & 4 ventral & $\underline{V A}, \underline{V B}$ & DD, VA, VD (1 posterior, 1 anterior), & AVEL, PVNR, VC01-03 & PVPR \\
\hline VB $(02,03,05,06)$ & 4 ventral & $\underline{D D}, V A, \underline{V D}$ & VB (1 posterior, ${ }^{a} 1$ anterior $\left.^{a}\right)$ & PVC & $\underline{A V B}$ \\
\hline$V^{*}(01,04,07)$ & 4 ventral & $\underline{2 \text { DD }}$ (1 posterior, 1 anterior), VA, VD & VB (posterior), $\mathrm{DB}^{a}$ & PVC & $\underline{A V B}$ \\
\hline VA $(02,04,06,07,08)$ & 4 ventral & 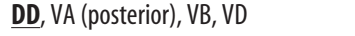 & $\underline{\mathrm{AS}}\left(\right.$ anterior $\left.^{a}\right)$ & $\underline{\text { AVA }}$, AVB, AVD, AVE & $\underline{A V A}, S A B D$ \\
\hline$V A^{*}(01,03,05)$ & 4 ventral & $\underline{2 \text { DD }}$ (1 posterior, 1 anterior), $\underline{\text { VD }}$ & $\underline{\text { AS }}$ (anterior $\left.{ }^{a}\right), \underline{D A}$ (posterior) & $\underline{\text { AVA }}$, AVD, AVE & $\underline{A V A}$, SABD \\
\hline
\end{tabular}

Motoneuron partners are at the same perimotor bin location unless mentioned anterior or posterior. Connections are from both left and right interneurons unless mentioned. Underlined are strong connections (2-10). Underlined and bold are stronger $(>10)$. VA and VB classes each has two subclasses marked with or without an asterisk.

${ }^{a}$ Three muscle lengths distance anterior or posterior.

Motoneuron class VC. Neuromuscular data were not available for the $\mathrm{VC}$ class of motoneurons. We referred to the original EM micrographs and notebooks of White et al. (1986), which cover the neuromuscular junctions of VC01, VC02, and VC03. We found that their neuromuscular junctions overlap and are sparser and cover more muscle cells than other ventral motoneurons. All three innervate ventral muscle cells 10, 9, 12, 11, and 14; VC01 and VC02 also innervate muscle cell 7, and only VC01 innervates muscle 8 (for muscle cell location, see Table 1 and Fig. 1a) (D. Hall and B. Chen, personal communication). The more posterior VC04 and VC05 innervate only vulva muscles while VC06 was not reconstructed. While it might be possible to find iterative connections for VC01-VC03 over the anterior portion, it will be impossible to extrapolate toward the posterior portion. We did not further analyze $\mathrm{VC}$ motoneurons and only their input to other motoneurons (mainly chemical synapses to DD and VD) is included in this model.

Iterativity index. To quantify the iterativity of connections, we devised an algorithm that describes each connection in relative terms along the perimotor AP axis and identifies connections that are present in multiple motoneurons of a given class. A single connection in each line of the published connectivity dataset is characterized by four values: source neuron $\mathrm{X}$, target neuron $\mathrm{Y}$, type of connection, and number found in EM micrographs. If $\mathrm{X}$ was a motoneuron, these data were extracted and kept for further analysis. If the target neuron $\mathrm{Y}$ was also a motoneuron or a muscle cell, the perimotor distance was calculated between the synaptic partners and was included in the name of the connection. To ensure that similar connections had similar designations, we expressed the perimotor distance in units of muscle bins that are 0.06 of the full-length perimotor space (see Results). Hence, for example, a chemical synapse from AS01 to VD01 that innervated directly opposing muscle cells (see Fig. $2 b$ ) was named "VD_S+00" ("VD" is the partner, "S" is for chemical synapse, and " +00 " for the perimotor distance; see Fig. 4) and similarly was named a chemical synapse from AS06 to VD06. By definition, the perimotor distance is positive if the partner is posterior to the motoneuron. For example, a gap junction from AS01 to VB03, which innervates posterior muscle cells (see Fig. $2 a$ ), was named "VA_E+02" ["VA" is the partner, "E" is for gap (electrical) junction, and " +02 " for two muscle bins, or three muscle lengths, posterior; see Fig. 4]. If the same name (in these examples, "VD_S+00" or "VA_E+02") was given to a number of connections above the iterativity threshold $(s)$, they were considered iterative.

To quantify the fraction of iterative connections present in the database, we defined an $I$ index $\left(I_{\mathrm{it}}\right)$ as follows:

$$
I_{\mathrm{it}}=\frac{\sum\left(\mathrm{Syn}_{\{>s\}}+\mathrm{GJ}_{\{>s\}}\right)}{\sum(\mathrm{Syn}+\mathrm{GJ})},
$$

where Syn and GJ are the total number of synapses and gap junctions, respectively, made to or from the specific motoneuron or class while $\operatorname{Syn}_{\{>s\}}$ and $\mathrm{GJ}_{\{>s\}}$ are the synapses and gap junctions that iterate more than the iterativity threshold. The iterativity index $\left(I_{\mathrm{it}}\right)$, therefore, represents the fraction of connections that iterate above a set threshold, among all connections made to and from a particular motoneuron class.

Determination of bin width for iterativity analysis. To determine iterativity of connection, we needed to evaluate their similarity to each other. To that end, we divided the continuous perimotor AP axis into discrete bins. However, the most suitable bin width was not known a priori. To identify the minimal bin width that would capture the majority of iterated connections at the best spatial resolution we plotted the iterativity index $I_{\mathrm{it}}$ for each class of motoneuron for different bin widths. We first calculated the mean distance occupied by a single muscle cell along the single-unit AP axis (defined as 0.0 to 1.0 from head to tail). The most anterior muscle cell is located at 0.15 (see Fig. $1 a$, "05") and the most posterior, 0.85 ("23"), with 19 muscle cells spanning the AP axis. The average normalized length of a muscle cell is therefore the following: $(0.85-0.15) / 19=0.04$. Plotted along bin width from one-half to three muscle lengths, $I_{\text {it }}$ was indeed sensitive to the spatial resolution used to calculate of the distance in perimotor space. $I_{\mathrm{it}}$, averaged among the classes remained almost constant as bin width was reduced from 0.12 (3 muscle lengths) to 0.06 ( 1.5 muscle lengths) but rapidly declined for bin widths $<0.06$. The values of average $I_{\text {it }}$ were $0.90 \pm 0.007$ (mean \pm SD) for a bin width of 0.06 or bigger, 0.85 for a bin width of 0.04 (one muscle length), and 0.72 when bin width was 0.02 . At bin widths $<0.06$, fewer types of iterative connections were present but the types of connections were not qualitatively different. We therefore used a bin width of 0.06 for further analysis.

Muscle rank as an alternative mapping method. The muscle cells are not perfectly uniformly distributed along the AP axis and are slightly more packed in the neck area than more posteriorly. We tested ranking the muscle cells as an alternative method to muscle length for mapping them in perimotor space. We ranked the muscle cells from anterior to posterior, making sure that opposing ventral and dorsal muscles have the same rank and used the rank instead of the location to calculate the functional location of each motoneuron. This is practically equivalent to measuring the body in muscle lengths that slightly increase along the body. The values of the iterativity indices $\left(I_{\mathrm{it}}\right)$ were 0.86 of all connections $(0.85$, $0.91,0.81,0.74,0.91,0.86$, and 0.85 for AS, DA, DB, DD, VA, VB, and $\mathrm{VD}$, respectively). Because these values are lower than those calculated using muscle length, we concluded that the ranking method misses some iterated connections that the muscle length formulation captures, and therefore used muscle length in the subsequent analysis.

Statistical evaluation of the iterativity index for individual motoneurons. We tested whether the level of iterativity observed in the connectivity dataset could arise at random. We performed a randomization test in which the resampled test networks were generated by shuffling the second column (the synaptic "target") of the connectivity dataset. The test networks retain the same number and types of connections for each motoneuron but randomize the targets of the connections. Hence, each observed value of $I_{\mathrm{it}}$ is compared with a population of values obtained 

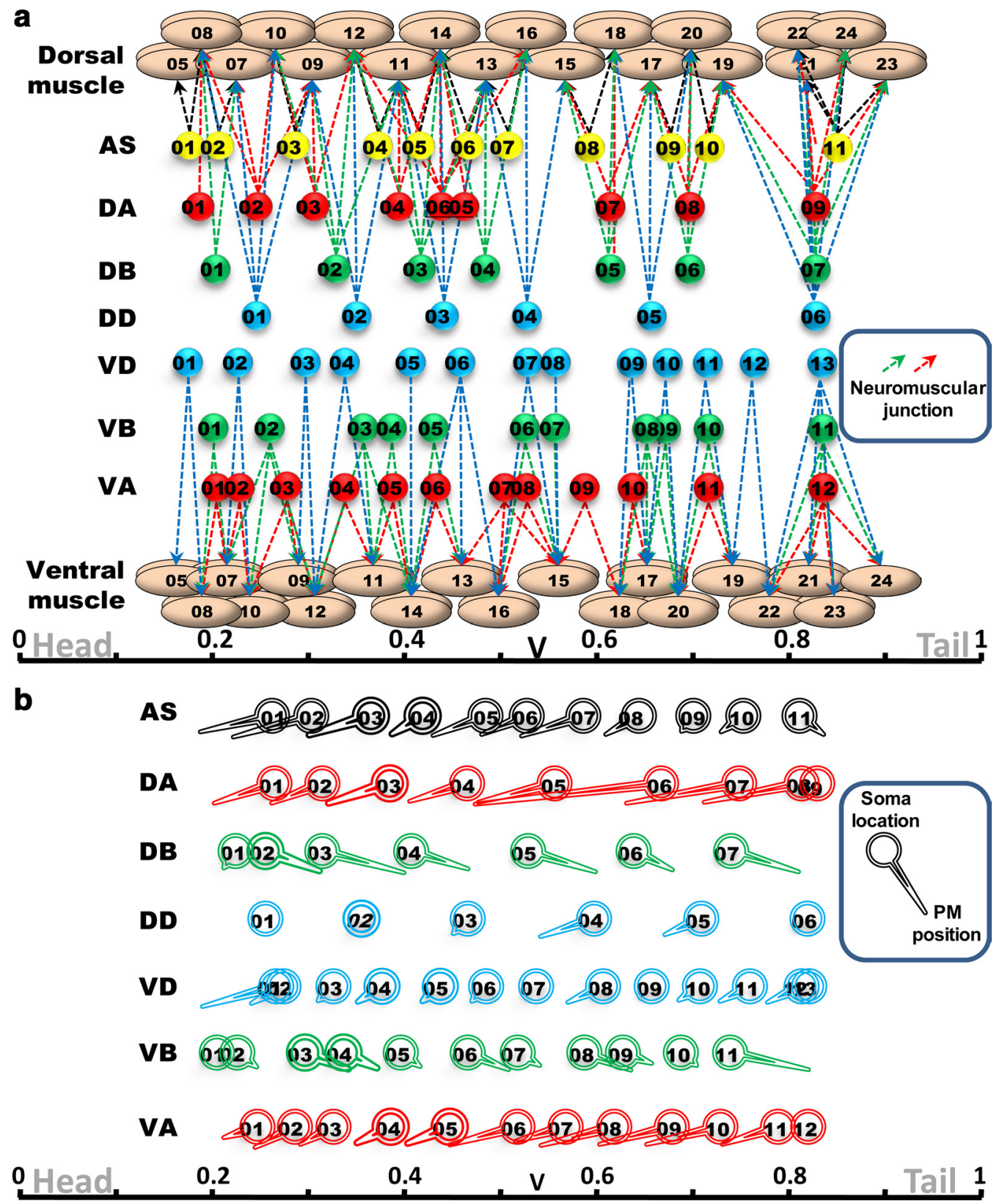

Figure 1. Remapping thelocomotion motoneurons and muscle cells in perimotor space. $\boldsymbol{a}$, Each dorsal (top) orventral (bottom) muscle cell ismapped in perimotor space atits anatomical AP location. Right and left paired muscle cells are presented for each location. Each motoneuron is mapped in perimotor space at the weighted average of AP locations of the muscle cells it innervates. The location of motoneuron classes along the dorsoventral axis is arbitrary and meant to facilitate visualization of connections and their relationships. $\boldsymbol{b}$, The perimotor location (empty circles) might not correspond to the anatomical location (tip of branches) of the cell body of each motoneuron. Notice that some motoneurons (e.g., DA06) move almost one-fifth of the length of the nematode anteriorly, while others move less, posteriorly, or not at all.

from the replicated test networks. The statistical significance of the difference between the actual and generated $I_{i t}$ indices can be then quantified using the standard nonparametric randomization test $p$ value as follows:

$$
p=\frac{1+\#\left\{I_{\mathrm{gen}}>I_{\mathrm{obs}}\right\}}{\mathrm{Nr}+1}
$$

where \#\{I $\left.I_{\text {gen }}>I_{\text {obs }}\right\}$ indicates the number of generated $I_{\mathrm{it}}$ values that are bigger than the observed value, and $\mathrm{Nr}$ is the number of generated networks. With only one animal sampled, it is not possible to compare the variability among our generated networks with the variability between real networks. This will only become possible when the networks of multiple nematodes have been reconstructed. We chose to use 500 shuffled networks $(\mathrm{Nr}=500)$ by plotting the $p$ values calculated for VD01VD07 along values of $\mathrm{Nr}$ from 50 to 1000 . The probability calculated for
VD01 was the most variable $(p=0.06$ at $\mathrm{Nr}=50)$ but together with the other motoneurons of its class converged toward the limit of the test $[1 /(\mathrm{Nr}+1)]$ and reached $p<0.01$ by $\mathrm{Nr} \geq 500$. Descriptive statistics are in the form (mean $\pm \mathrm{SE}, \mathrm{SD}$ ), unless otherwise stated.

Cluster analysis. We used hierarchical clustering (MATLAB Statistics Toolbox function: linkage) to evaluate whether some of the classes of motoneurons can be further subdivided. We computed the Euclidean distance between pairs of objects in the matrix that represent each motoneuron in a class and the connections it makes (presented as color maps in Fig. 4) and creates an agglomerative hierarchical cluster tree from the distances in the matrix. We then constructed clusters from the tree. We set a limit of two clusters using the "distance" criterion. The function finds the smallest height at which a horizontal cut through the tree leaves two or one clusters. Classes that formed two distinct clusters were considered to have two subclasses (marked with asterisks in Figs. 6 and 7 and Table 2). 

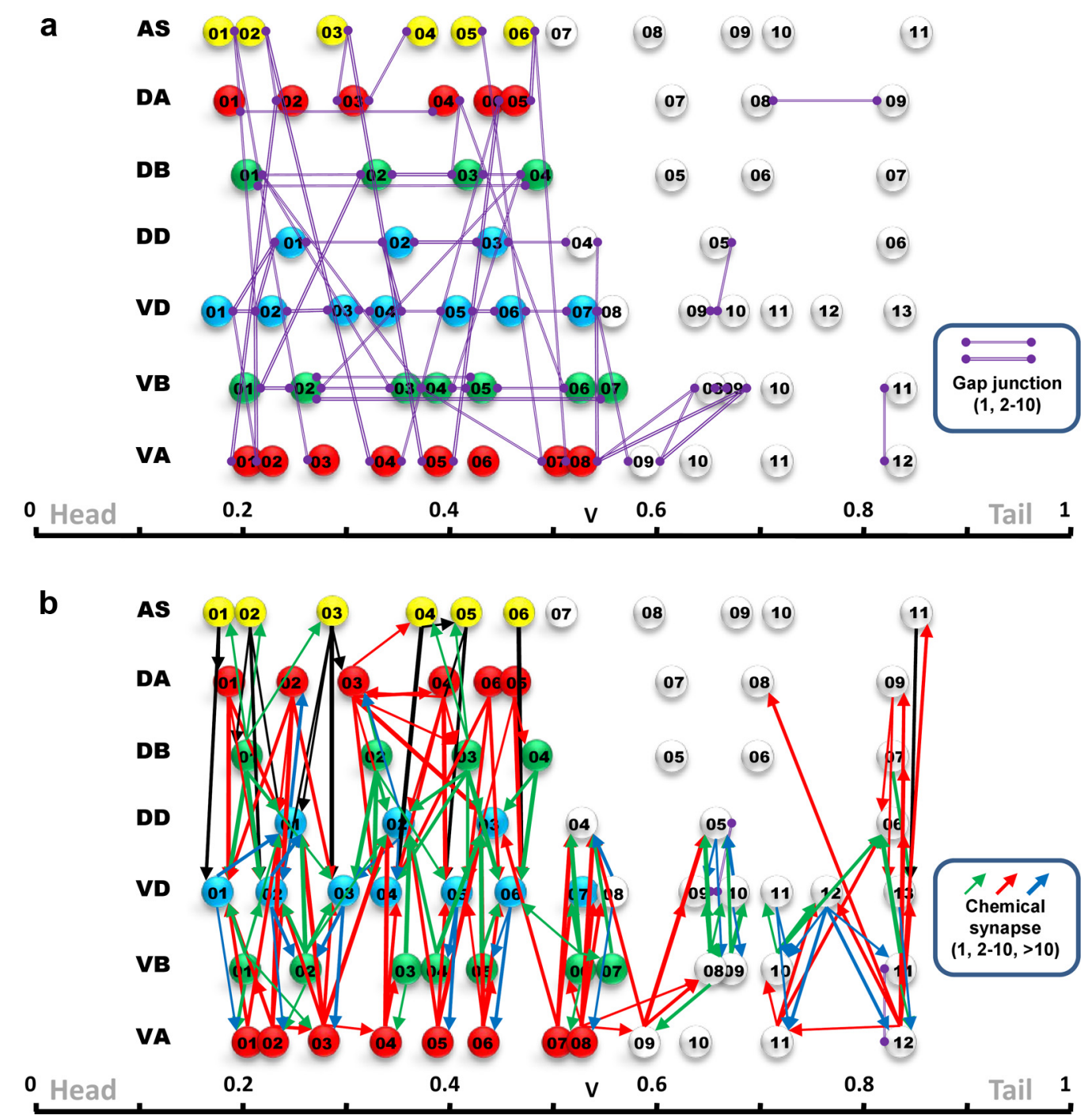

Figure 2. Remapping connections among motoneurons in perimotor space. All electrical (round ends) ( $\boldsymbol{a}$ ) and chemical (arrows) (b) synapses among motoneurons in the dataset are depicted in perimotor space. As in Figure 1, the motoneuron position along the AP axis is determined by the innervated muscle cells and the dorsoventral axis is arbitrary to facilitate visualization. More specifically, iterating connections are parallel to each other in this diagram. Motoneuron classes and their outgoing chemical synapses are color-coded (AS, yellow/black; DA and VA, red, DB and VB, green; DD and VD, blue), while those with partial or no connectivity information are left colorless.

\section{Results}

Remapping of muscle and motoneurons in perimotor space We defined a "perimotor space" in which elements of the network (motoneurons and muscle cells) are positioned by their function during locomotion. The conventional indexing of $C$. elegans muscle cells does not describe their location because it is derived from cellular lineage. Hence, the order of dorsal muscle cells is as follows: $2,1,4,3,6,5,8,7,10,9,12,11,14,13,16,15,18$, $17,20,19,22,21,24$, and 23 (Fig. 1a). The two dorsal and two ventral quadrants of muscle cells are laterally symmetric in position and innervation. In an undulating animal such as the nematode, the function of a muscle is determined by its location on the anteriorposterior (AP) (head-to-tail) and transverse axes (in the nematode, this axis indicates whether a muscle is dorsally or ventrally located). We mapped muscle cells in perimotor space (Fig. 1a, Table 1) so that $\mathrm{PM}_{n}$, their perimotor position is their anatomical midpoint between anterior and posterior extremities of the sarcomere region (Dixon and Roy, 2005), projected onto the AP axis [data file NeuronFixedPoints.xls from the study by Chen et al. (2006)]. For example, dorsal muscle cell $10\left(\mathrm{PM}_{10}=0.25\right)$ is between muscle cells $07\left(\mathrm{PM}_{07}=\right.$ $0.21)$ and $09\left(\mathrm{PM}_{09}=0.29\right)$.
It follows that the function of a motoneuron is determined by the collective functions of the muscles it innervates. We defined the position of each motoneuron in perimotor space $\left(\mathrm{PM}_{\mathrm{MN}}\right)$ as the mean of the perimotor positions of the muscle cells it innervates, weighted by the anatomical size (number observed) of each neuromuscular junction as follows:

$$
\mathrm{PM}_{\mathrm{MN}}=\frac{\sum_{n=1}^{N}\left(\mathrm{PM}_{n} \times \mathrm{NMJ}_{n}\right)}{\sum_{n=1}^{N} \mathrm{NMJ}_{n}},
$$

where $\mathrm{PM}_{\mathrm{MN}}$ is the calculated perimotor position of the motoneuron, $N$ is the number of innervated muscle cells, $\mathrm{PM}_{n}$ is the perimotor position of an innervated muscle cell, and $\mathrm{NMJ}_{n}$ is the anatomical size of the neuromuscular junction. We chose this formulation to reflect the functional action of a motoneuron rather than simply the mean of the perimotor position of the innervated muscle cells. For example, the motoneuron AS01 (Fig. $1 a$, top left) is positioned at 0.155 in perimotor space because it innervates dorsal muscle cells MD05 (AP position, 0.15; NMJ size, 3.25) and MD08 (AP position, 0.16; NMJ size, 3.25). The muscle cells send arms that originate perpendicularly from the 
middle third of each muscle fiber and connect to motoneurons that have a region of neuromuscular junction at that location on the ventral or dorsal nerve cord (Dixon and Roy, 2005; Altun and Hall, 2008b). We hence accepted the assumption made by Chen et al. (2006) that each muscle cell is postsynaptic to all motoneurons that have a neuromuscular junction along its middle third and used their designation of neuromuscular junctions. The muscle cells are arranged as almost symmetric left-right pairs. Because of the designation method and the left-right symmetry of the muscle cells, each muscle cell assumed to be innervated on the right is always also assumed to be innervated on the left. Hence, we only included this information once and used the right muscle cell of each pair. It is important to note that the location of a motoneuron in perimotor space does not necessarily correspond to the actual anatomical location of its soma (Fig. 1b, Table 1; soma location data from WormAtlas/NeuronType.csv and B. Chen, personal communication). In the most extreme example, DA06 is more anterior than DA05 in perimotor space.

The perimotor distance $\left(\mathrm{PD}_{x y}\right)$ is defined as the difference between the perimotor positions $\left(\mathrm{PM}_{x}\right.$ and $\left.\mathrm{PM}_{y}\right)$ of the motoneurons $x$ and $y$. All interconnections among motoneurons in the dataset, comprising 119 gap junctions and 1119 chemical synapses, are mapped in perimotor space in Figure 2, $a$ and $b$, respectively. Sensory and interneurons are not mapped in perimotor space because they do not innervate muscle. Connections to and from those neurons (1658 gap junctions and 5275 chemical synapses) are used in the analysis described below but for reasons of clarity are not shown in Figure 1. In this diagram, the dorsal muscle cells and motoneurons are on top, while their ventral counterparts are on the bottom. Only one of the two laterally symmetric quadrants is presented for the dorsal and only one for the ventral sides. The distribution of motoneuron classes along the vertical axis is arbitrary and meant to facilitate visualization of the connections. The perimotor framework allows direct functional interpretation of the diagram. Thus, motoneurons and muscle cells that are positioned at the same $\mathrm{AP}$ position $\left(\mathrm{PD}_{x y}=0\right)$, for example, AS02, DB01, and dorsal muscle cell 08 are all in the same perimotor position. A positive perimotor distance $\left(\mathrm{PD}_{x y}>0\right)$ indicates that the second motoneuron (for example, DD02) innervates muscle cells that are posterior to those innervated by the first motoneuron (for example, AS03).

Four main observations arise from this novel description of the motoneuronal connectivity dataset: (1) Most connections are local in the perimotor space $(98 \%$ are made within a $\mathrm{PD}<0.25$; see Fig. 3). The exceptions are gap junctions within motoneurons of the DA and the DB classes, with the largest PD of 0.295. (2) The sparse data for motoneuron connectivity posterior to the midpoint (location of the vulva marked by a "V" at 0.54 in Fig. 2) are qualitatively different from the anterior data. As explained above, electron micrographs of this region in a hermaphrodite nematode were not taken, and these data are partial and from a male nematode (White et al., 1986; Chen et al., 2006; Varshney et al., 2011) (D. Hall, personal communication). (3) The connectivity of the most anterior motoneurons differs from the other motoneurons. This may be due to an edge effect, their different morphology (White et al., 1976, 1986), or both. (4) Using the perimotor formulation, we found that many connections iterate. By "iterate," we mean that motoneurons of the same class make connections with neurons of a similar class at similar perimotor distances. For example, AS01 is presynaptic to VD01, and AS02 is presynaptic to VD02, and so on for all ASs and VDs. Similarly,

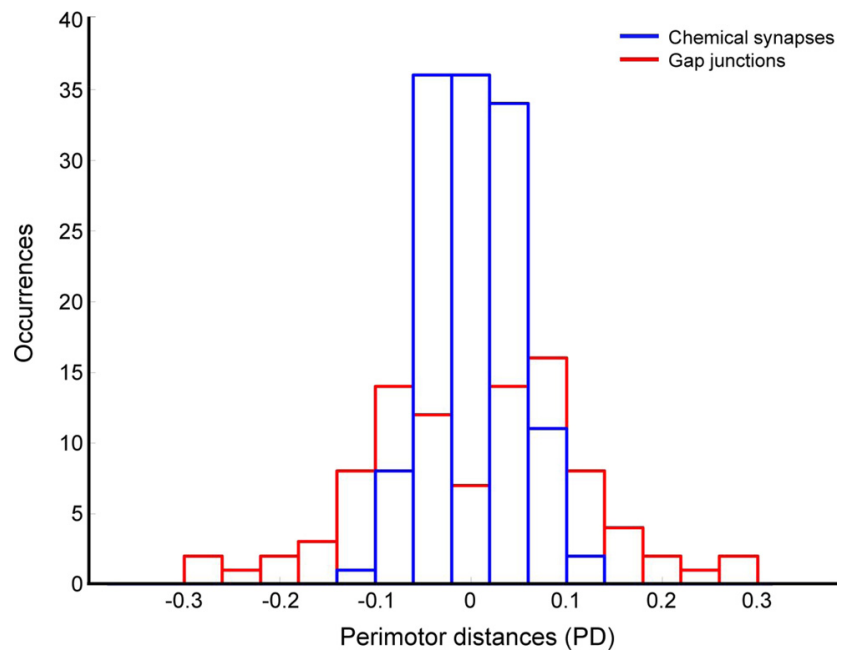

Figure 3. Distribution of perimotor distances. The PD for each chemical synapse (blue bars) and gap junction (red bars) in the dataset was calculated as the difference between the position in perimotor space $\left(\mathrm{PM}_{\mathrm{MN}}\right)$ of the partners. The bins of the histogram are 0.04 , which correlate to the mean estimated length of a muscle cell (see Materials and Methods). Most (98\%) connections are local ( $P D<0.25$ ). More specifically, $84 \%$ of chemical synapses (blue bars) are to motoneurons that innervate the same muscle cell or an adjacent neighbor, and $82 \%$ of gap junction (red bars) are to motoneurons that innervate the same muscle cell or up to three muscle cell lengths in either direction.

DD01 makes a gap junction onto DD02, which in turn makes a similar connection to DD03.

\section{Iterative connections in the dataset}

When the iterativity threshold was two (two or more similar connections defined as iterating), 0.9 of the total connections were included (iterativity indices $I_{\mathrm{it}}=0.90,0.90,0.89,0.91,0.93$, 0.90 , and 0.88 for AS, DA, DB, DD, VA, VB, and VD, respectively; Fig. 4). Thus, the great majority of connections made by each class of motoneuron occur more than once. If we restrict the analysis to connections that iterate three or more times, then $82 \%$ of the connections are included $\left(I_{\text {it }}=0.81,0.86,0.72,0.85,0.90\right.$, 0.76 , and 0.82 for $\mathrm{AS}, \mathrm{DA}, \mathrm{DB}, \mathrm{DD}, \mathrm{VA}, \mathrm{VB}$, and $\mathrm{VD}$, respectively). We did not examine connections that iterate four or more times because this would have exceeded the number of reconstructed DD motoneurons and therefore excluded them from the analysis.

\section{Anterior-posterior distribution of iterativity among individual motoneurons}

We next calculated the $I_{\text {it }}$ for each individual motoneuron (Fig. 5, solid blue dots), in contrast to the previous analysis, which was performed on each class of motoneuron. For an individual motoneuron, $I_{\mathrm{it}}$ is the fraction of connections that iterate among all its connections. We found that at an iterativity threshold of two, the $I_{\text {it }}$ was $>0.7$ for most motoneurons ( 39 of 41 ) from the neck to the vulva (mean $\pm \mathrm{SE}, 0.9 \pm 0.02 ; \mathrm{SD}, 0.12$ ). The two outliers (range, $0.44-0.62)$ are located in the neck area $(0.16-0.19$ on the perimotor AP axis).

To establish whether these iterativity indices could be generated by random connectivity, we generated and analyzed multiple test networks by shuffling the second column (the synaptic "target") of the original dataset. The test networks retained the same number and type of connections for each motoneuron but randomized the synaptic targets. We generated 500 randomized 
VA 0.93

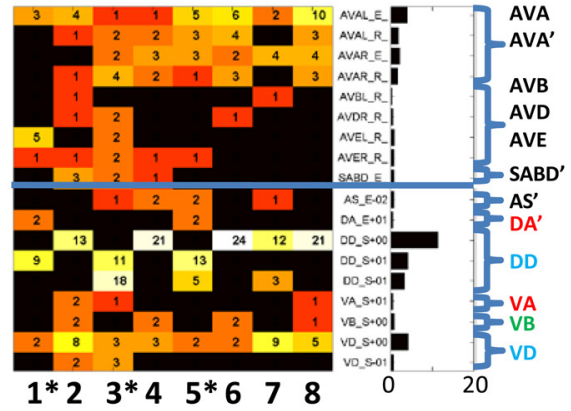

DA 0.90

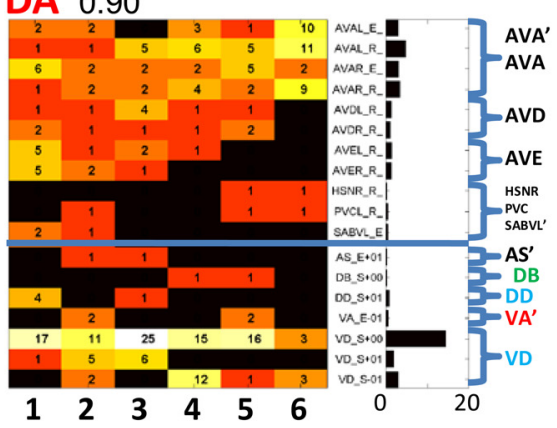

AS 0.90

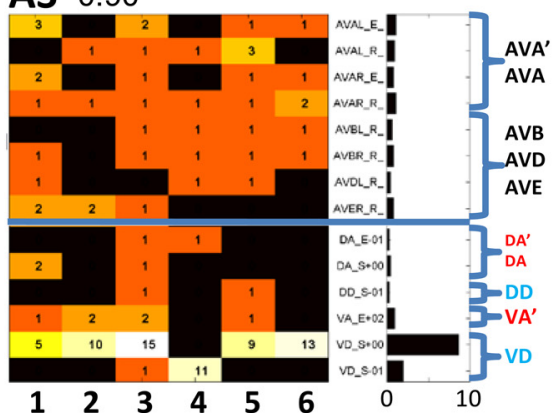

VB 0.90

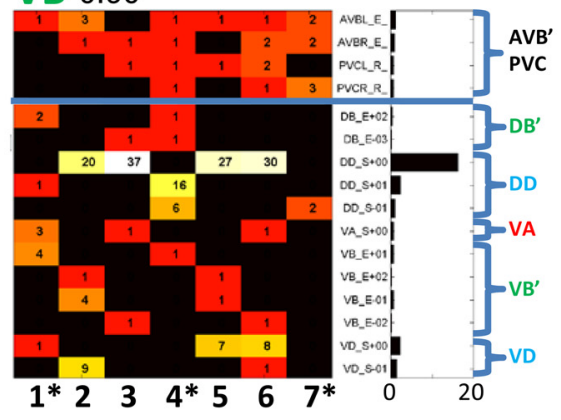

DB $\quad 0.89$

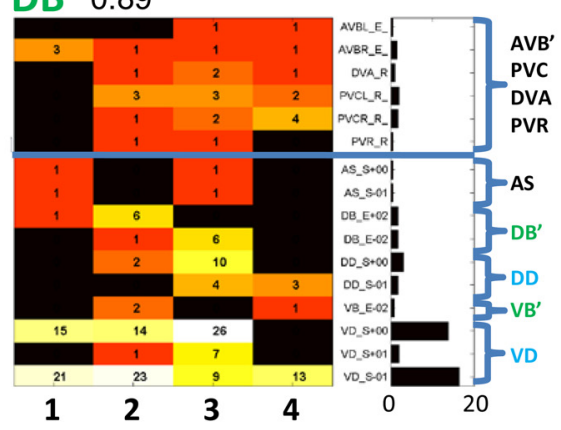

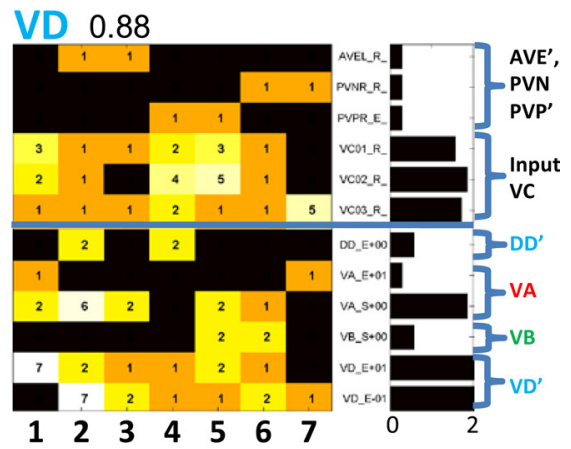

DD 0.91

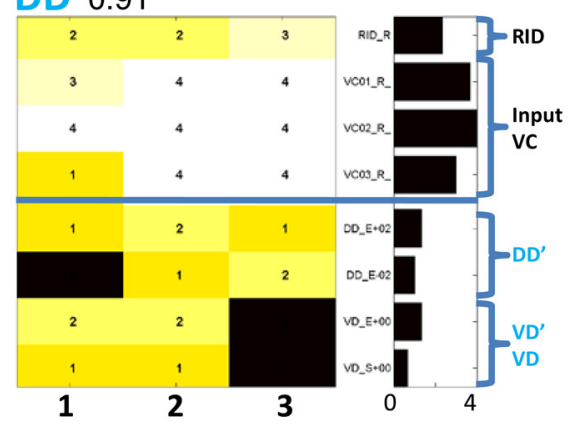

Figure 4. Analysis of iterative connections. Iterativity analysis of the synaptic connections of each class of motoneuron (named at top left corner of each panel) is represented as a color map and a bar graph. Color maps (black, 0 connections; red, lowest value, to white, highest value) are arranged such that individual numbered motoneurons within the class are columns, while rows represent types of iterative connections. The numbers in each box represent the number of anatomical connections made by the source motoneuron represented by the column (e.g., VA1) to the iterative connection represented by the row. The iterativity threshold is set to two. That is, a connection is iterative if two or more members of the source class make synaptic connections to neurons of the same perimotor distance and class. The structure of connection names (e.g., "VD_S-1"; a chemical synapse to a VD motoneuron anterior to the source motoneuron) are described in the main text. The bars to the right of each panel represent the average number of synapses made by each cell in the source class onto the target motoneuron (below blue horizontal line) or input from another neuron (above line). Connection descriptions to the right of the bar graphs are simplified and motoneuron names are color-coded (e.g., "AVA" means chemical synapse from the interneuron AVA; "AVA" " means gap junctions from same). Iterativity index for each class is indicated next to class name (e.g., 0.93 for VA). Numbers with asterisks represent subclasses within VA and VB as described in the main text and Figure 6.

networks and calculated the $I_{i t}$ indices for each individual motoneuron (Fig. 5, open red circles). The average $I_{i t}$ for this randomized population was $0.42 \pm 0.01, \mathrm{SD}=0.09$, and each value was significantly smaller than the actual $I_{i t}$ of each motoneuron ( $p<0.05$ for VD01 and VB01; $p<0.01$ for all other motoneurons). We conclude that the occurrence of iterating connections is significantly different from that of random networks. The gap in connectivity data of the posterior portion of the locomotor network could be filled, without further assumptions, by iterating each of these connections among the motoneuron in their calculated perimotor locations. To address more general rules of organization, we chose to pursue the hypothesis that the occurrence of iterating connections reflects the existence of a core set of muscle cells and motoneurons together with their interconnections that repeats along the AP axis.

\section{Motoneurons stereotypic of each class compose a repeating segment}

The existence of iterated connections that are characteristic of each motoneuron class (Table 2, Fig. 4) allowed us to construct stereotypical motoneurons representing each class (Fig. 6). This analysis revealed that every stereotypical motoneuron innervates two or three muscle cells and is connected by up to three chemical synapses and two or three gap junctions with other motoneurons. Excitatory motoneurons make their strongest chemical synapses to the opposing inhibitory motoneuron or motoneurons (VD, DD). Excitatory motoneurons also receive chemical synapses from interneurons while inhibitory motoneurons receive mostly synapses from the three anterior VC motoneurons. Motoneurons of the classes $\mathrm{DB}, \mathrm{DD}, \mathrm{VB}$, and VD have gap junctions with an anterior and a posterior member of their class. Five classes (AS, 


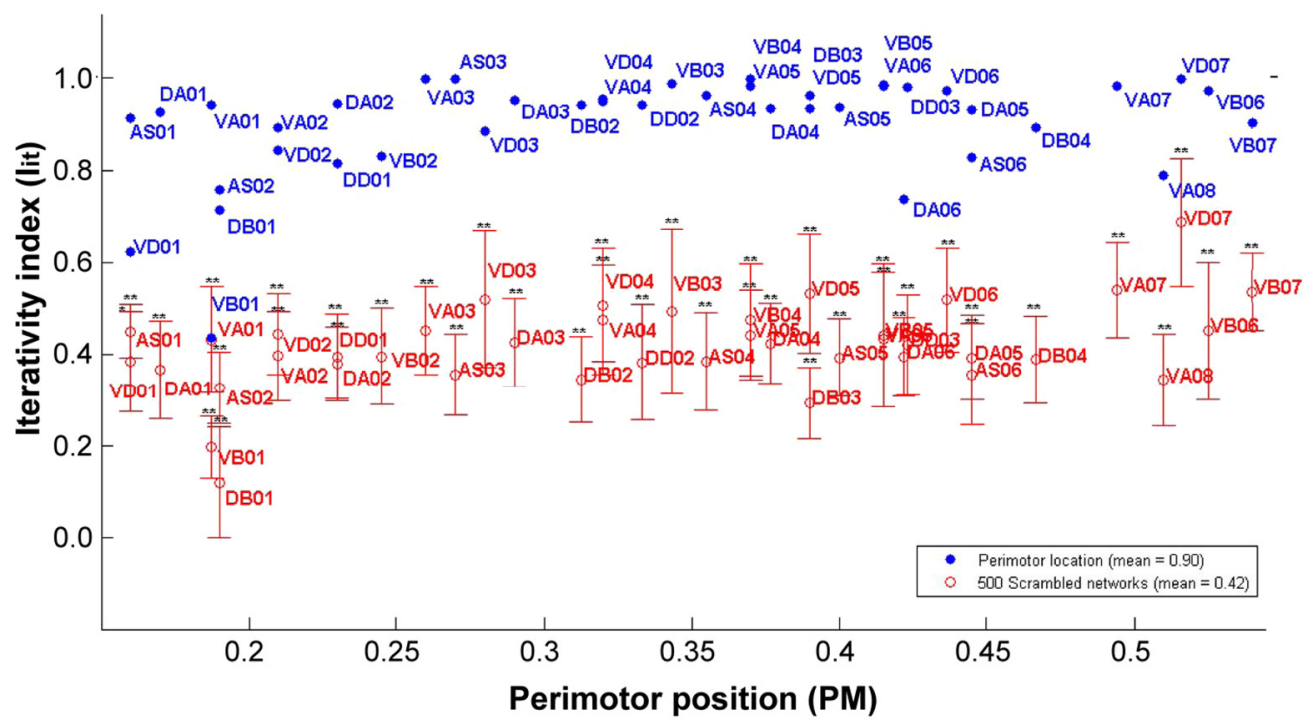

Figure 5. Distribution of iterativity along AP axis and statistical validation. The iterativity index $\left(l_{i t}\right)$ of each individual motoneuron in the dataset (solid blue dots) is plotted along the AP axis $(0.0-1.0)$ together with the distribution of the same quantity in 500 generated networks (open red circles) in which the target neurons were randomly shuffled. The dataset values are higher and significantly different from the generated population $\left({ }^{*} p<0.05 ;{ }^{* *} p<0.01\right.$ ). We decided to use 500 scrambled networks by plotting (inset) the value of $p$ for each VD motoneuron (VD01 to VD07) against the number of generated networks ( $\mathrm{Nr}$ ) from 50 to 1000. The value of $p$ varies at low $\mathrm{Nr}$ but converges to the limit of the statistical test $[1 /(\mathrm{Nr}+1)$; thick gray line] by $\mathrm{Nr}=500$.

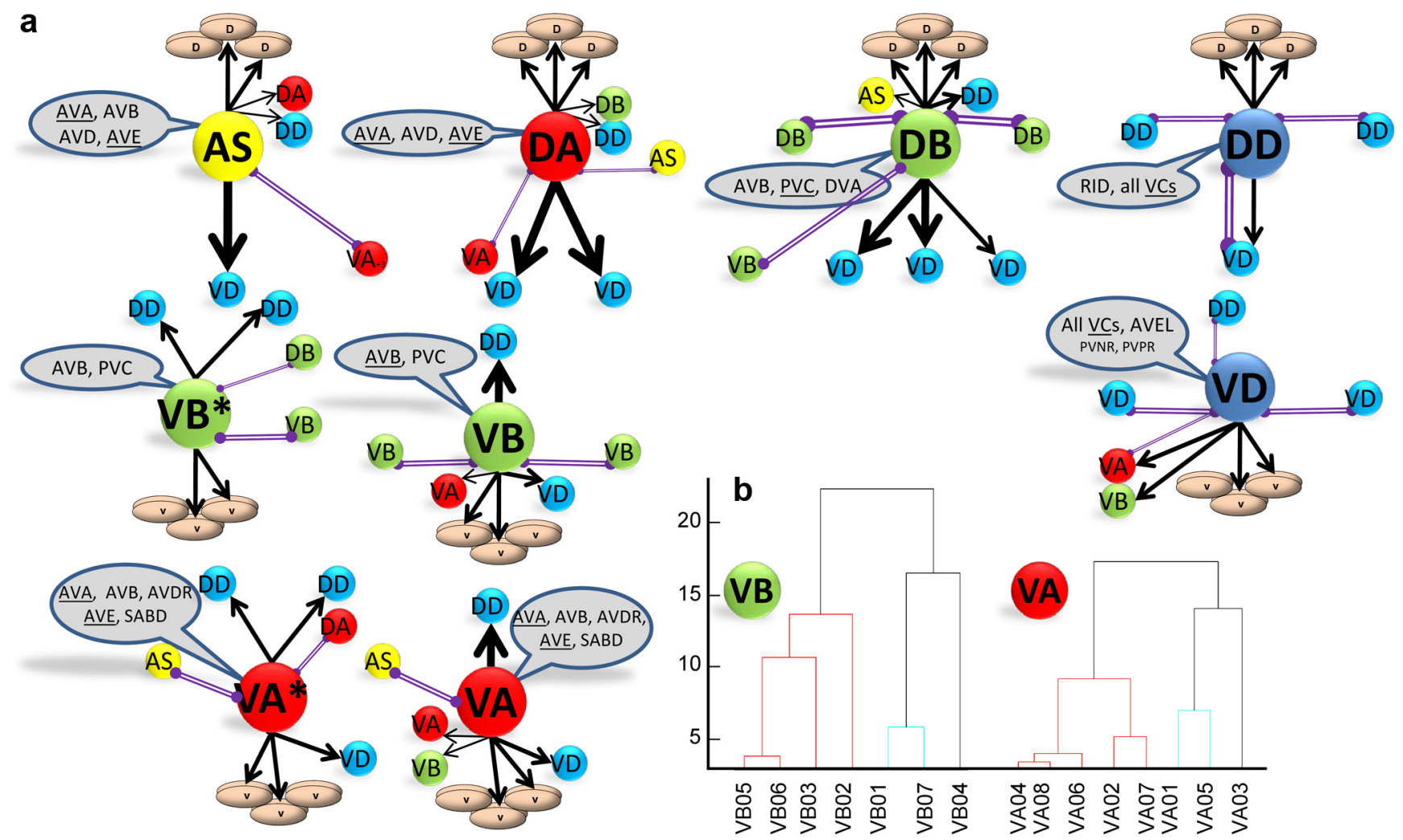

Figure 6. Stereotypic motoneurons. $\boldsymbol{a}$, The iterating connections for each class of motoneurons are shown as black arrows (chemical, directional, synapses) or purple double lines (bidirectional gap junctions). $\boldsymbol{b}$, Cluster analysis was used to investigate whether the five more numerous classes (AS, DA, VA, VB, and VD) could be subdivided further. Of those, VB and VA (presented) could be further divided: VB01, VB04, and VB07 (VB*) and similarly VA01, VA03, and VA05 (VA*) have slightly different connectivity than their counterparts. These motoneurons are indicated by asterisk here and in Figures 3 and 6.

$\mathrm{DA}, \mathrm{VA}, \mathrm{VB}$, and VD) have almost twice as many members as the other three (DB, DD, and VC). We examined whether these five classes could be further divided into subclasses. For this purpose, we used hierarchical clustering on the set of iterating connections of each class and determined that VA and VB indeed segregate into subclasses with subtle differences, while DA, VD, and AS do not (Fig. 6b). The same subclasses are evident whether the iterativity threshold was two or three.

We next constructed a wiring diagram of a minimal circuit containing at least one member of each class and the muscle fiber they innervate. To ensure that the complement of motoneurons in this minimal circuit reflected those of the whole population, 
a

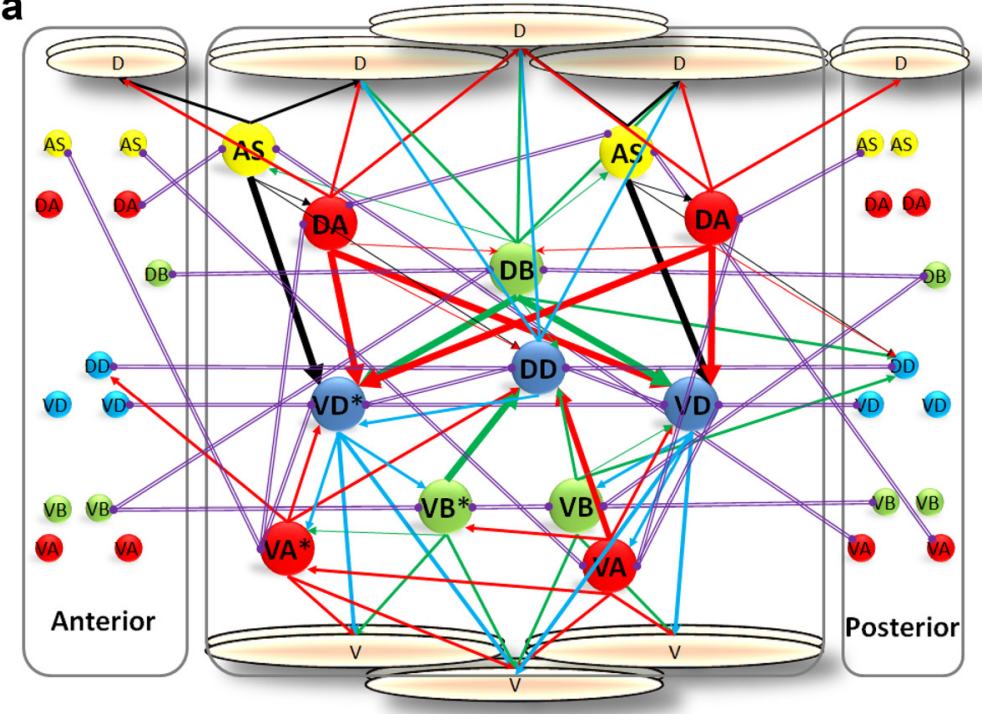

b

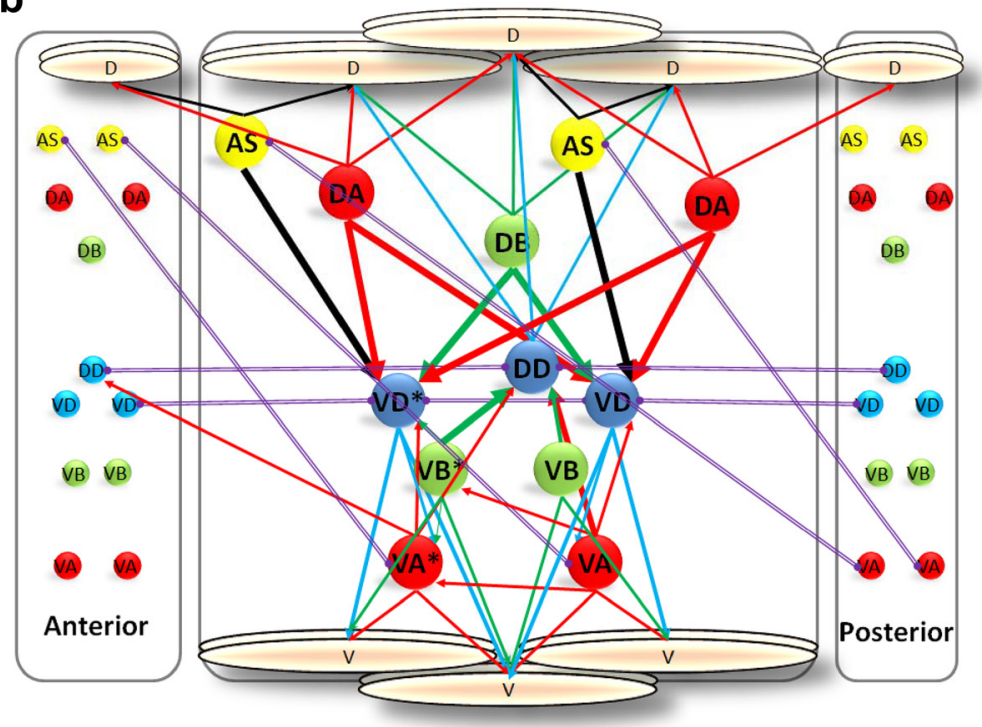

Figure 7. A segment composed of stereotypic motoneurons. Twelve stereotypic motoneurons [iterativity threshold of $2(\boldsymbol{a})$ or $3(\boldsymbol{b})]$ and six muscle cells compose a segment. Each segment contains all iterating neuromuscular junctions (arrows to muscle cells), chemical synapses (arrows, colored as source and thickness proportionate to number of synapses in the data set), and gap junctions (purple double lines, size proportionate to number of gap junctions in the data set). Inputs from sensory and interneurons are included in the model but not presented. To facilitate visualization, some motoneurons are not at their perimotor location within the segment.

we used a single $\mathrm{DD}$ and $\mathrm{DB}$ motoneuron and pairs of the remaining motoneurons (AS, DA, VB, VA). Therefore, the perimotor model segment includes 12 muscle cells and 12 motoneurons: six dorsal muscle cells co-innervated by two AS, two DA, and one of each DB and DD motoneurons and six opposing ventral muscle cells, innervated by two of each VA, VB, and VD motoneurons. The model segment includes all interconnectivity among the motoneurons, the connections to the anterior and posterior segments and the input from sensory and interneurons (Fig. 7, not presenting the latter).

This framework provided novel insights into the network organization that would be difficult to obtain with alternative representations. For example, (1) gap junctions connect every $\mathrm{DB}, \mathrm{VB}, \mathrm{DD}$, and $\mathrm{VD}$ to their anterior and posterior neighbors of the same class. In contrast to previously published results (Sengupta and Samuel, 2009), other motoneuron classes (AS, VA, and DA) are not chained in this fashion. (2) Gap junctions also connect each AS to a VA in a more posterior segment. (3) A pair of AS motoneurons innervates a pair of VD motoneurons that inhibit the opposing ventral muscles. (4) Each DA receives chemical synapses from an AS that innervates the same dorsal muscle cells and innervates two VD motoneurons that innervate the opposing ventral muscle cells. (5) Chemical synapses connect each DB to the two VD motoneurons that innervate the opposing ventral muscle cells and posterior DD motoneurons. Each DB also has a gap junction with an anterior VB. (6) Each VD motoneuron innervates two muscle cells and the VB and VA motoneurons that innervate them.

\section{Connectivity model}

By repeating and interconnecting this minimal perimotor segment six times along an AP axis, we could construct a connectivity model of the full motoneuronal network (Fig. 8a). Three segments can be demarcated within the span of the observed connectivity dataset (Fig. $8 b, c$ ), supporting the repeating unit hypothesis. These segments in the original dataset are similar to the equivalent segments in the connectivity model but also include some noniterating connections (Fig. 9). We set out to compare the segmentation of the model with that of the dataset. A commonly used measure of the balance between local and remote connections is modularity. We followed the definition of modularity $(Q)$ provided by Newman and Girvan (2004). To calculate Q, neurons and muscle cells were considered as vertices and synapses and gap junctions as edges. Modularity was defined (Newman and Girvan, 2004) as the fraction of the edges in the network that connect vertices of the same community (segment) minus the expected value of the same quantity in a network with the same community (segmental) divisions but random connections between the vertices.

We defined $Q$ as follows:

$$
Q=\frac{E_{\mathrm{w}}}{E_{\mathrm{w}}+E_{\mathrm{b}}}-\frac{\left(V_{\mathrm{w}}-1\right)}{\left(V_{\mathrm{w}}+V_{\mathrm{b}}-1\right)},
$$

where $Q$ is the modularity measure, $E_{\mathrm{w}}$ and $E_{\mathrm{b}}$ are the number of edges within and between modules, respectively, and $V_{\mathrm{w}}$ and $V_{\mathrm{b}}$ are the number of vertices in the same or in other modules, respectively. Modularity values for networks with strong community structure typically fall in a range of $\sim 0.3$ to 0.7 (Newman and Girvan, 2004). In our model, each segment contains 30 local synapses and 7 gap junctions. Each segment (except from the posterior and anterior-most segments) also shares 16 gap junctions with other segments, sends 4 synapses to, and receives an equal number of synapses from the neighboring segments. Hence, each segment 
a

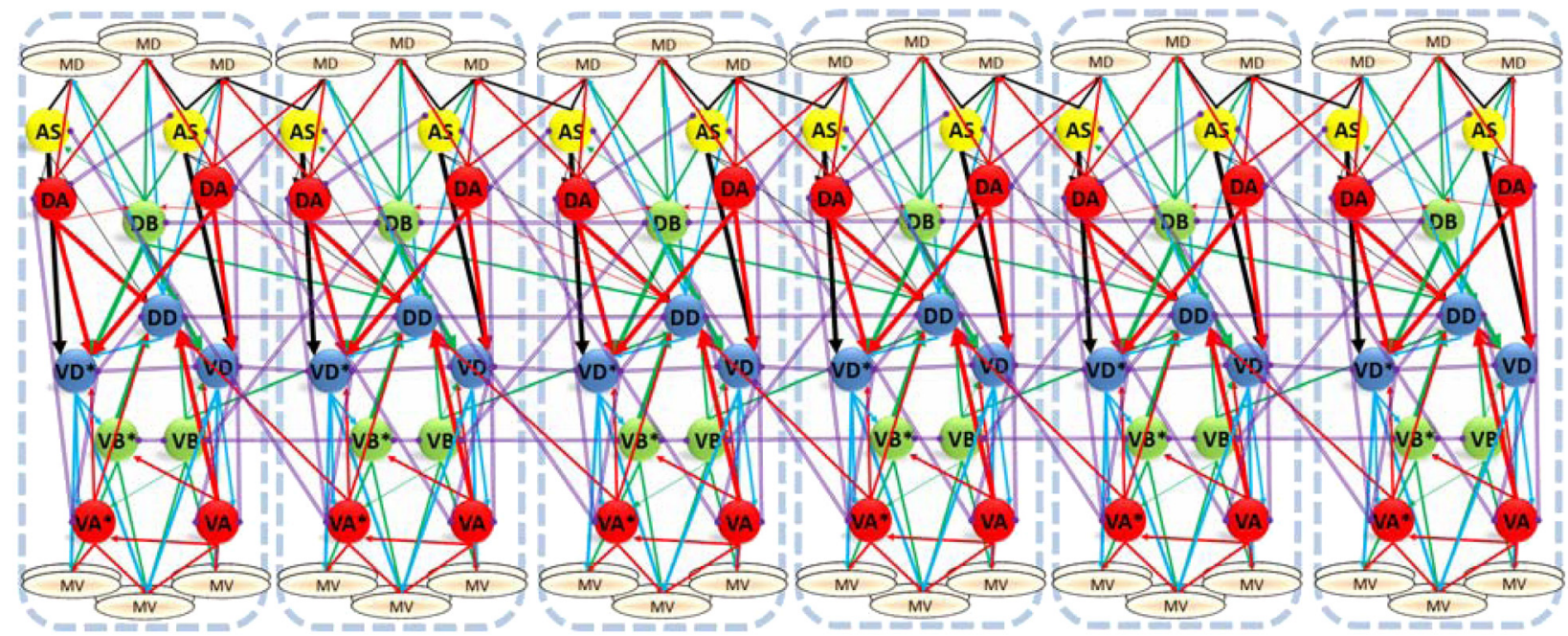

b
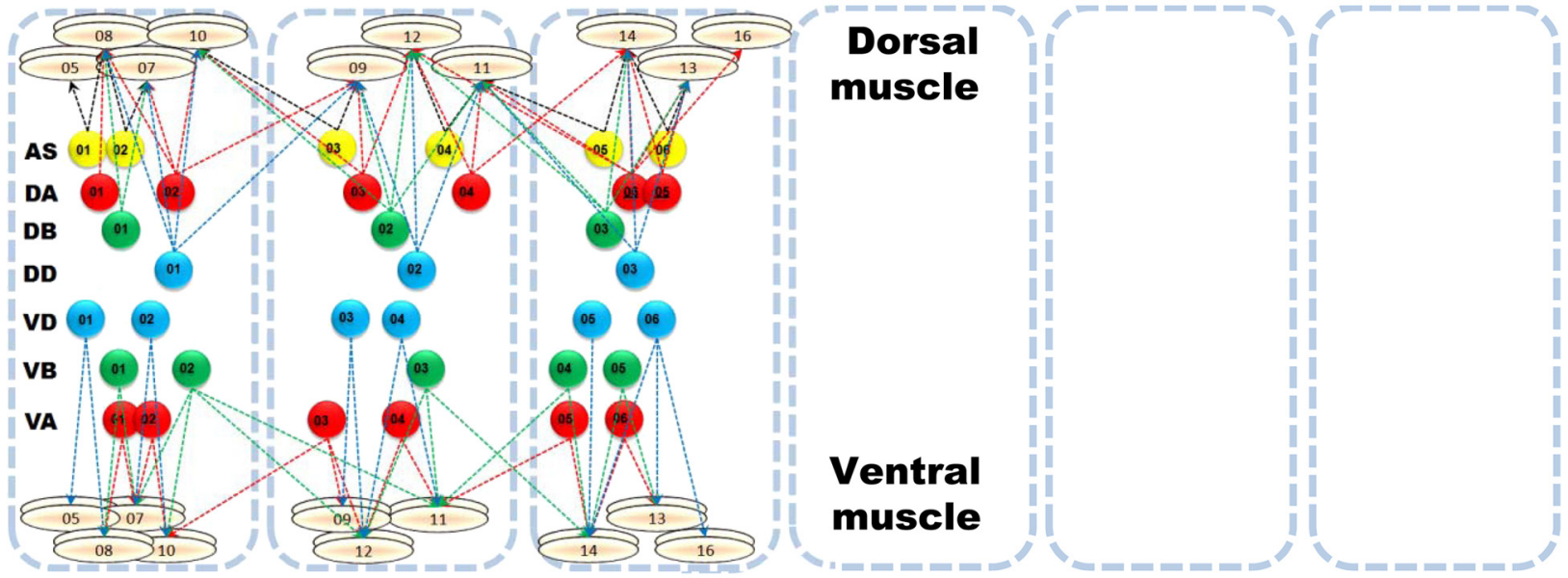

C
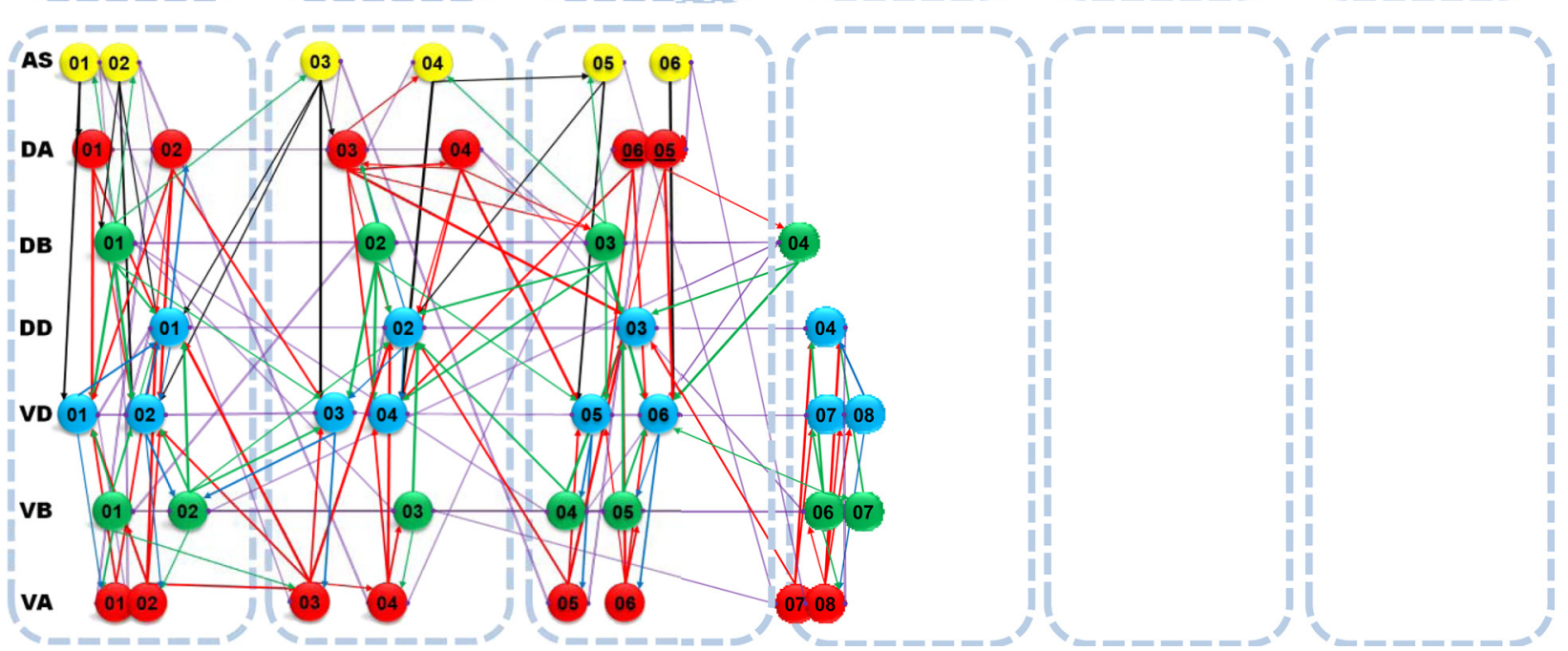

Figure 8. Perimotor segments in dataset and connectivity model. $\boldsymbol{a}$, Six segments can be interconnected along an AP axis to produce a complete connectivity model of the locomotion network (threshold of two is presented). $\boldsymbol{b}, \boldsymbol{c}$, When motoneurons, muscle cells, neuromuscular junctions (b), and interneuronal connections (c) are presented in perimotor framework, three segments emerge from the partial dataset. Small gaps were inserted between the segments to facilitate visualization of the segments (compare with Figs. 1 and 2). The complete motoneuronal system is expected to span six segments (empty frames).

includes $37(30+7)$ edges among motoneurons and $20(4+16)$ edges connect adjacent segments (Fig. 7). In a randomly connected network, each pair of vertices (neurons) has an equal chance of connecting. We excluded the possibility of selfconnecting because it does not appear in the data. Hence, the ratio between possible intrasegmental partners (11) and total possible partners (71) can be used as the expected fraction of the edges in the network that connect vertices of the same community. This gives calculated $Q$ values of $0.53=\left(37^{\star} 6 /\left(37^{\star} 6+\right.\right.$ $\left.\left.20^{\star 5}\right)-11 / 71\right)$ when iterativity threshold $(s)$ was set to two. When iterativity threshold was set to three, $Q$ was $0.58=\left(21^{\star} 6 /\right.$ $\left.\left(21^{\star} 6+9{ }^{\star} 5\right)-11 / 71\right)$. Because the existing connectivity data- 


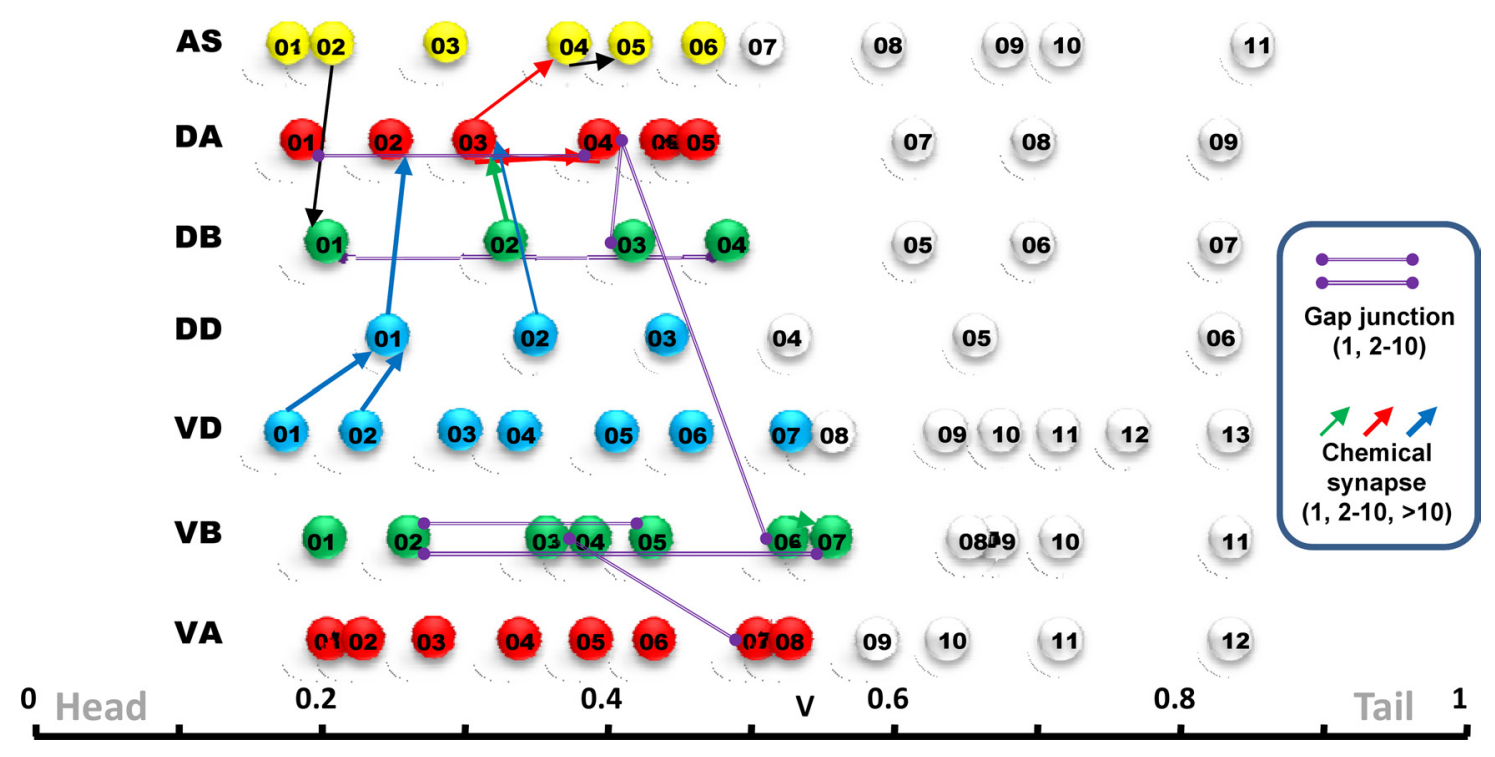

Figure 9. The noniterating connections among motoneurons. Seven electrical (round ends) and 11 chemical (arrows) synapses among motoneurons in the dataset are not iterating (compare with Fig. 2 and Table 2). Connections among and with the motoneurons with partial or no connectivity data were omitted as they were not included in the analysis.

set spans only the anterior half of the nerve cord, we identified only three segments in the connectivity dataset (Fig. $8 b, c)$. We then followed the same procedure to calculate modularity and calculated a $Q$ value of 0.37 . This measure of modularity is an underestimate because the additional segments provide targets for the randomized network that are included in the denominator of $Q$ equation. When additional three segments are included with the same mean number of connections and motoneurons the value of $Q$ is 0.48 . These relatively high $Q$ values support the hypothesis of a repeating core set of muscle cells and motoneurons. The complete connectivity database of $C$. elegans was previously calculated to have a $Q$ of 0.66 (Kashtan and Alon, 2005; corrected $Q_{\mathrm{m}}$ of 0.52). For our and for formerly published calculation of modularity, edges were considered undirected and unweighted.

\section{Discussion}

\section{Perimotor space}

Many studies equate the location of a neuron to the anatomical location of its cell body (Ahn et al., 2006; Chen et al., 2006; Kaiser and Hilgetag, 2006; Varier and Kaiser, 2011). In fact, for most neurons with extensive dendritic trees, the location of the cell body provides little information about the function of the neuron. Our goal in the present work was to find a mapping or transformation that would facilitate the identification of patterns of connectivity. We reasoned that functionally related groups of motoneurons would be defined by the muscles they innervate. Because the muscles are arrayed approximately linearly along the AP axis of the nematode, it seemed reasonable to array the motoneurons along this axis. By expressing the position of other motoneurons relatively, according to this scheme, we were able to group connections according to their function. These coordinates chart a new framework, the perimotor space. It is important to realize that the mapping of function onto a linear array corresponding to muscle location is one of many ways to order and segregate functionally related connections. Unlike the nematode, where the location of a muscle defines its function, in jointed limbs the relationship between the location of a muscle and its mechanical action is more complex. However, an analogous approach to the mapping used here would be to map the position of motoneurons in perimotor space along a normalized temporal or phase axis according to the activity of the motoneurons during a single cycle of locomotion (Yakovenko et al., 2002). The concept of perimotor space we used here shares some similarities with myotopic neuronal maps described for the motoneuron dendrites of Drosophila larvae (Landgraf et al., 2003; Dixit et al., 2008). However, we find perimotor space more useful because it incorporates the function of the muscles and hence allows direct behavioral interpretation of the model. Furthermore, perimotor mapping can provide the functional meaning and interpretability of network structure when coupled with network analysis using graph theory (Bullmore and Sporns, 2009) for existing and novel wiring datasets. In such analysis, nodes that are directly involved in locomotion (motoneurons or specific areas of the CNS) will be fixed in perimotor space. One system that might benefit from this type of analysis is a single segment of the mammalian spinal cord.

The perimotor description of the motoneuronal network of $C$. elegans (Fig. 2) has revealed a hitherto-undescribed modularity in the synaptic connections of motoneurons. Furthermore, it supports some existing hypothesis while generating new ones. For example, two motoneuron classes, AS and DA, were known to be morphologically similar except for the presence of a long, nonsynapsing dorsal neurite directed anteriorly in DA but not AS motoneurons (White et al., 1976). In the present work, we have shown that they also have different synaptic connections and hence are likely to serve different roles in the locomotor circuit. Specifically, each DA makes neuromuscular junctions to three muscles and synaptic connections to two VD motoneurons, while each AS innervates two muscle cells and only one VD. AS motoneurons have gap junctions with VA motoneurons in a posterior location and to an anterior DA, while DA motoneurons have gap junctions with VA motoneurons that innervate the opposing muscle cells (Fig. 7). In addition, AS motoneurons are innervated by both AVA and AVB interneurons and therefore might be activated during both forward and backward locomotion, while DA are innervated by AVA, AVD, and AVE (Chalfie et al., 1985; White et al., 1986; present study) and are activated during backward but not forward locomotion (Haspel et al., 
2010) and are necessary for backward locomotion (Chalfie et al., 1985).

\section{Iterative connections}

Once we generated the motoneuronal network in perimotor space, we were able to detect iterative patterns within the connections. Most connections made by motoneurons of the same class are with neurons of a similar class at similar perimotor distances. This iterativity is not apparent when only the anatomical locations of the neurons are considered. Some of the connections that iterate are anatomically scarce and were not considered important before. For example, each AS motoneuron has a single gap junction to a VA motoneuron that innervates ventral muscle cells posterior to those innervated by the former.

Two previously used assumptions are crucial for our analysis to be valid and meaningful. First, the single individual organism from which data were gathered should have stereotypical connectivity for the species, sex, and developmental stage. Second, the pattern of connectivity of the posterior half should be similar to that of the anterior half. If, following the acquisition of new connectivity data, either assumption is proven wrong, the wiring diagram of a segment may have to be altered. However, the validity of the method of analysis will still hold and new data can be easily incorporated to produce a more refined model of a segmental wiring diagram. In addition, as described above, we adopted assumptions regarding the neuromuscular innervations previously made by Chen et al. (2006).

A periodically repeated sublineage was reported for five classes of motoneurons (VA, VB, VC, VD, and AS) (White et al., 1976), which we suspected might underlie the perimotor iterativity. However, when we compared the cellular lineage (Sulston et al., 1983; http://www.wormbase.org/db/searches/pedigree) to the perimotor and anatomical locations (Fig. 1b), we found that the cellular lineage is closely related to the location of the cell bodies along the ventral cord but not to the function of the motoneuron or its perimotor location.

The analysis of iterative connectivity emphasizes connections based on their similarity among modules and eliminates connections that iterate below an arbitrary threshold (we used the most conservative threshold of two connections). It can be applied to many segmental networks. Furthermore, it could be modified to accommodate other modular networks (in which modules are similar to each other), for example, the connectivity within and among cortical columns. In this case, the analysis will have to be modified to include a second dimension.

\section{Perimotor segmentation and the connectivity model}

Segmentation is very prevalent among undulatory motor systems (Puhl and Mesce, 2010) such as leech and lamprey. Moreover, undulatory propagation was recently suggested to underlie activity in the mammalian spinal cord during motor behavior (Cuellar et al., 2009). The study of the relatively small motoneuronal network underlying undulatory locomotion in C. elegans as a segmented network might reveal fundamental principles shared with these more complex systems. Segmentation of the motoneuronal network was suggested for another nematode species, Ascaris lumbricoides (Stretton et al., 1978; Davis and Stretton, 1996), in which six segments contain 11 motoneurons each. The segmentation was based on the anatomical location of the commissures along the body and soma location of the motoneurons. However, the pattern of commissures along $C$. elegans body is different and does not suggest a repeating unit, and the adult $C$. elegans has not been considered to be segmented (Minelli and
Fusco, 2004). By keeping only connections that iterate in the same class of motoneurons, we defined the stereotypical motoneuron of each class. We then assembled a perimotor segment, which we iterated six times to produce the connectivity model. Most of the chemical synapses (27 of 33) in this model are within segments, while most of the gap junctions (14 of 20) are between segments. Gap junctions have been suggested before to be important for the coordination of motor output (Kiehn and Tresch, 2002). There are several important ways in which this connectivity model differs from formerly published models. Mainly, it is completely based on the currently available neuroanatomical dataset. We did not arbitrarily choose only motoneurons and connections that fit a specific hypothesis of their role in locomotion. Rather, the connectivity model includes all motoneuron classes and iterating connections. The VC class of motoneurons is included as input to other motoneurons as explained in Materials and Methods.

Advances in automation and novel neuroanatomical tools are generating a growing number of connectivity datasets (Micheva and Smith, 2007; Lu et al., 2009; Katz et al., 2010). The representation of such datasets is a crucial step toward gaining insight into the principles that underlie neuronal connectivity. Published representations have commonly used parameters of connectivity derived from the data itself to localize nodes in dimensionless space (such as clustering and attraction/repulsion algorithms) or along axes of such parameters (such as eigenvalues, adjacency, or hierarchy). These representations emphasize specific features of the network but do not reflect the function of the network. The perimotor representation we introduce is based on the function of muscle and the pattern of motoneuronal innervation. We have used it to discover and extend rules of connectivity in the data available for the anterior portion of $C$. elegans locomotion network and construct a novel, segmented, connectivity model. The perimotor space is not limited to this network but can be generalized and extended to connect graph theory analysis with network function in other networks.

\section{References}

Ahn YY, Jeong H, Kim BJ (2006) Wiring cost in the organization of a biological neuronal network. Physica A 367:531-537.

Altun ZF, Hall DH (2008a) Neuronal wiring. In: WormAtlas. Available at http://www.wormatlas.org/neuronalwiring.html.

Altun ZF, Hall DH (2008b) Handbook of C. elegans anatomy. In: WormAtlas. Available at http://www.wormatlas.org/hermaphrodite/ hermaphroditehomepage.htm.

Bassett DS, Greenfield DL, Meyer-Lindenberg A, Weinberger DR, Moore SW, Bullmore ET (2010) Efficient physical embedding of topologically complex information processing networks in brains and computer circuits. PLoS Comput Biol 6:e1000748.

Bryden JA, Cohen N (2004) A simulation model of the locomotion controllers for the nematode Caenorhabditis elegans. In: Proceedings of the Eighth International Conference on the Simulation of Adaptive Behavior (Schaal S, Ijspeert AJ, Billard A, Vijayakumar S, Hallam J, Meyer JA, eds), pp 183-192. Cambridge, MA: MIT Press.

Bryden J, Cohen N (2008) Neural control of Caenorhabditis elegans forward locomotion: the role of sensory feedback. Biol Cybern 98:339-351.

Bullmore E, Sporns O (2009) Complex brain networks: graph theoretical analysis of structural and functional systems. Nat Rev Neurosci 10:186-198.

Chalasani SH, Chronis N, Tsunozaki M, Gray JM, Ramot D, Goodman MB, Bargmann CI (2007) Dissecting a circuit for olfactory behaviour in Caenorhabditis elegans. Nature 450:63-70.

Chalfie M, White J (1988) The nervous system. In: The nematode Caenorhabditis elegans (Wood WB, ed), pp 337-391. Cold Spring Harbor, NY: Cold Spring Harbor Laboratory.

Chalfie M, Sulston JE, White JG, Southgate E, Thomson JN, Brenner S (1985) The neural circuit for touch sensitivity in Caenorhabditis elegans. J Neurosci 5:956-964. 
Chen BL, Hall DH, Chklovskii DB (2006) Wiring optimization can relate neuronal structure and function. Proc Natl Acad Sci USA 103:4723-4728.

Cuellar CA, Tapia JA, Juárez V, Quevedo J, Linares P, Martínez L, Manjarrez E (2009) Propagation of sinusoidal electrical waves along the spinal cord during a fictive motor task. J Neurosci 29:798-810.

Davis RE, Stretton AO (1996) The motornervous system of Ascaris: electrophysiology and anatomy of the neurons and their control by neuromodulators. Parasitology 113:S97-S117.

Dixit R, Vijayraghavan K, Bate M (2008) Hox genes and the regulation of movement in Drosophila. Dev Neurobiol 68:309-316.

Dixon SJ, Roy PJ (2005) Muscle arm development in Caenorhabditis elegans. Development 132:3079-3092.

Gray JM, Hill JJ, Bargmann CI (2005) A circuit for navigation in Caenorhabditis elegans. Proc Natl Acad Sci U S A 102:3184-3191.

Haspel G, O'Donovan MJ, Hart AC (2010) Motoneurons dedicated to either forward or backward locomotion in the nematode Caenorhabditis elegans. J Neurosci 30:11151-11156.

Kaiser M, Hilgetag CC (2006) Nonoptimal component placement, but short processing paths, due to long-distance projections in neural systems. PLoS Comput Biol 2:e95.

Karbowski J, Schindelman G, Cronin CJ, Seah A, Sternberg PW (2008) Systems level circuit model of $C$. elegans undulatory locomotion: mathematical modeling and molecular genetics. J Comput Neurosci 24:253-276.

Kashtan N, Alon U (2005) Spontaneous evolution of modularity and network motifs. Proc Natl Acad Sci U S A 102:13773-13778.

Katz PS, Calin-Jageman R, Dhawan A, Frederick C, Guo S, Dissanayaka R, Hiremath N, Ma W, Shen X, Wang HC, Yang H, Prasad S, Sunderraman R, Zhu Y (2010) NeuronBank: a tool for cataloging neuronal circuitry. Front Syst Neurosci 4:9.

Kiehn O, Tresch MC (2002) Gap junctions and motor behavior. Trends Neurosci 25:108-115.

Landgraf M, Jeffrey V, Fujioka M, Jaynes JB, Bate M (2003) Embryonic origins of a motor system: motor dendrites form a myotopic map in Drosophila. PLoS Biol 1:E41.

Lu J, Tapia JC, White OL, Lichtman JW (2009) The interscutularis muscle connectome. Plos Biol 7:e32.

Micheva KD, Smith SJ (2007) Array tomography: a new tool for imaging the molecular architecture and ultrastructure of neural circuits. Neuron 55:25-36.

Milo R, Shen-Orr S, Itzkovitz S, Kashtan N, Chklovskii D, Alon U (2002) Network motifs: simple building blocks of complex networks. Science 298:824-827.
Minelli A, Fusco G (2004) Evo-devo perspectives on segmentation: model organisms, and beyond. Trends Ecol Evol 19:423-429.

Morita S, Oshio K, Osana Y, Funabashi Y, Oka K, Kawamura K (2001) Geometrical structure of the neuronal network of Caenorhabditis elegans. Physica A 298:553-561.

Newman ME, Girvan M (2004) Finding and evaluating community structure in networks. Phys Rev E Stat Nonlin Soft Matter Phys 69:026113.

Niebur E, Erdös P (1991) Theory of the locomotion of nematodes: dynamics of undulatory progression on a surface. Biophys J 60:1132-1146.

Niebur E, Erdös P (1993) Theory of the locomotion of nematodes: control of the somatic motor neurons by interneurons. Math Biosci 118:51-82.

Puhl JG, Mesce KA (2010) Keeping it together: mechanisms of intersegmental coordination for a flexible locomotor behavior. J Neurosci 30:2373-2383.

Qian J, Hintze A, Adami C (2011) Colored motifs reveal computational building blocks in the C. elegans brain. PLoS One 6:e17013.

Sengupta P, Samuel AD (2009) Caenorhabditis elegans: a model system for systems neuroscience. Curr Opin Neurobiol 19:637-643.

Sporns O, Kötter R (2004) Motifs in brain networks. PLoS Biol 2:e369.

Stretton AO, Fishpool RM, Southgate E, Donmoyer JE, Walrond JP, Moses JE, Kass IS (1978) Structure and physiological activity of the motoneurons of the nematode Ascaris. Proc Natl Acad Sci U S A 75:3493-3497.

Sulston JE, Schierenberg E, White JG, Thomson JN (1983) The embryonic cell lineage of the nematode Caenorhabditis elegans. Dev Biol 100:64-119.

Varier S, Kaiser M (2011) Neural development features: spatio-temporal development of the Caenorhabditis elegans neuronal network. PLoS Comput Biol 7:e1001044.

Varshney LR, Chen BL, Paniagua E, Hall DH, Chklovskii DB (2011) Structural properties of the Caenorhabditis elegans neuronal network. PLoS Comput Biol 7:e1001066.

Von Stetina SE, Treinin M, Miller DM 3rd (2006) The motor circuit. Int Rev Neurobiol 69:125-167.

Watts DJ, Strogatz SH (1998) Collective dynamics of "small-world" networks. Nature 393:440-442.

White JG, Southgate E, Thomson JN, Brenner S (1976) The structure of the ventral nerve cord of Caenorhabditis elegans. Philos Trans R Soc Lond B Biol Sci 275:327-348.

White JG, Southgate E, Thomson JN, Brenner S (1986) The structure of the nervous system of the nematode Caenorhabditis elegans. Philos Trans R Soc Lond B Biol Sci 314:1-340.

Yakovenko S, Mushahwar V, VanderHorst V, Holstege G, Prochazka A (2002) Spatiotemporal activation of lumbosacral motoneurons in the locomotor step cycle. J Neurophysiol 87:1542-1553. 